\title{
Variante de colgajo de rotación tipo Hamstring para reconstrucción de úlcera isquiática
}

\author{
Hamstring rotation flap variant for ischial ulcer reconstruction
}

\author{
José Israel Espino-Gaucín", Armando Guillermo Apellaniz Campo², Carlos Altamirano Arcos', Luciano \\ Nahas Combina1, Enrique Chávez Serna³ ${ }^{3}$ Edgar Vargas Flores¹, Carlos Rodríguez Rodríguez ${ }^{1}$
}

\begin{abstract}
RESUMEN
Antecedentes. El colgajo Hamstring es una opción válida para la reconstrucción de úlceras isquiáticas. Las úlceras por presión representan un verdadero desafío para el cirujano plástico. Requieren un tratamiento especializado y multidisciplinario por su alta probabilidad de recidiva y las complicaciones en su manejo. Por lo general, ocurren por presión sostenida sobre la tuberosidad isquiática.

Caso clínico. Se presenta el caso de un paciente parapléjico desde el nacimiento secundario a mielomeningocele con diagnóstico actual de úlcera isquiática. Se le realizaron durante 3 años múltiples intentos de cierre quirúrgico alternando medidas conservadoras, sin éxito. A la exploración física presenta una úlcera isquiática izquierda de $6 \times 6 \mathrm{~cm}$ a la que se le realizó aseo y desbridación quirúrgica más cierre por medio de un colgajo Hamstring modificado. A los 7 días presentó dehiscencia de herida la cual se manejó de manera conservadora y cierre primario diferido a las 4 semanas. A 8 meses de posoperatorio el paciente se encuentra con buena evolución y con resultado estético y funcional aceptable, por otra parte, se encuentra sin recidiva, lo que ha permitido su reinserción laboral y un buen equilibrio físico-psico-emocional. Conclusión. El objetivo de este colgajo es brindar cobertura al área isquiática sin afectar estructuras adyacentes, lo cual requiere de un amplio conocimiento de la anatomía de la región femoral, así como de los colgados tanto locales como regionales que podemos utilizar para la reconstrucción. Actualmente este colgajo es una opción terapéutica efectiva para el tratamiento de úlceras isquiáticas refractarias en centros que cuentan con cirujanos plásticos no especializados en microcirugía.
\end{abstract}

Palabras claves: colgajo Hamstring, úlcera isquiática.

\begin{abstract}
Background. The Hamstring flap is an adequate option for ischial ulcers reconstruction. Pressure ulcers represent a real challenge for the plastic surgeon. They require specialized and multidisciplinary treatment due to their high recurrence probability and complications in their management. Regularly, pressure ulcers occur when sustained pressure is applied on the ischial tuberosity.

Case report. We report a case of a 23-year-old paraplegic male with myelomeningocele complaining for an ischial ulcer. Multiple attempts to surgical closure were made during three years along with conservative management, without success. Physical examination revealed a $6 \times 6 \mathrm{~cm}$ left ischial ulcer, which was managed with surgical debridement and closure with a modified Hamstring flap. After 7 days, the patient presented wound dehiscence, which was managed conservatively and primary delayed closure after 4 weeks. 8 months postoperatively the patient has a good evolution and an acceptable functional and aesthetic result, without recurrence, which has allowed his reintegration into work and a good physical-psycho-emotional balance.

Conclusion. The objective of this flap is to provide coverage to the ischial area without affecting adjacent structures, which requires a broad knowledge of the anatomy of the femoral region, as well as the local and regional flaps that can be used for reconstruction. Nowadays this flap is an effective therapeutic option for the treatment of refractary ischial ulcers in plastis suergery centers without microsurgery.
\end{abstract}

Key words: Hamstring flap, ischiatic ulcer.

REVISTA ARGENTINA DE CIRUGÍA PLÁSTICA 2020;26(1):41-44. HTTPS://DOI.ORG/10.32825/RACP/202001/0041-0044

\section{INTRODUCCIÓN}

Las úlceras isquiáticas (UI) son una problemática a nivel mundial en pacientes con lesiones de la médula espinal, presentando aumento en la carga física y financiera, así como son una causa de hospitalización no planificada ${ }^{1}$. Las úlceras isquiáticas (UI) ocurren por aumento de presión por períodos prolongados en el sitio de la tuberosidad isquiática ${ }^{2}$. Existen otros factores agregados como humedad, desnutrición, hipoperfusión, pero los que se reportan como los principales factores para la producción de la UI son la falta de sensibilidad y la inmovilidad ${ }^{3}$.

1. Residente de cirugía plástica. Hospital General “Manuel Gea González". México, D.F

2. Médico adscrito de Cirugía Plástica. Hospital General "Manuel Gea González". México, D.F

3. Médico Interno de Pregrado. Hospital General Manuel Gea González. México, D.F

$\triangle$ Correspondencia: José Israel Espino Gaucin. Cordles@hotmail. com, Israel.espino@gmail.com

Los autores no declaran conflictos de intereses

Recibido: 21/06/2019 / Aceptado: 30/08/2019
Los pacientes más afectados son aquellos con lesión medular, y es una de las causas más importantes de morbilidad 4 . Así como se ha reportado mayor tasa de complicaciones y recurrencia en este tipo de pacientes. Por esta razón, cuando se ofrece una cobertura se deben tener en cuenta los factores de riesgo y se deben implementar estrategias de reducción de riesgo cuando se implemente el tratamiento quirúrgicos.

El tratamiento de las úlceras isquiáticas requieren un equipo especializado, así como un manejo multidisciplinario para promover una cicatrización completa, con cobertura total y buena perfusión ${ }^{6}$. Por esta razón se deben tener en cuenta los conceptos de éxito para el tratamiento en las UI, los cuales son:?

1. Resección de tejidos desvitalizados.

2. Bursectomía completa.

3. Osteotomía y moldeamiento.

4. Cobertura con adecuado relleno y aporte sanguíneo.

A la fecha, existen varios colgajos miocutáneos y fasciocutáneos que se han utilizado en la reconstrucción de las $\mathrm{UI}^{8}$. Estas opciones reconstructivas están basadas principalmente en los músculos glúteo mayor, gracilis, ten- 


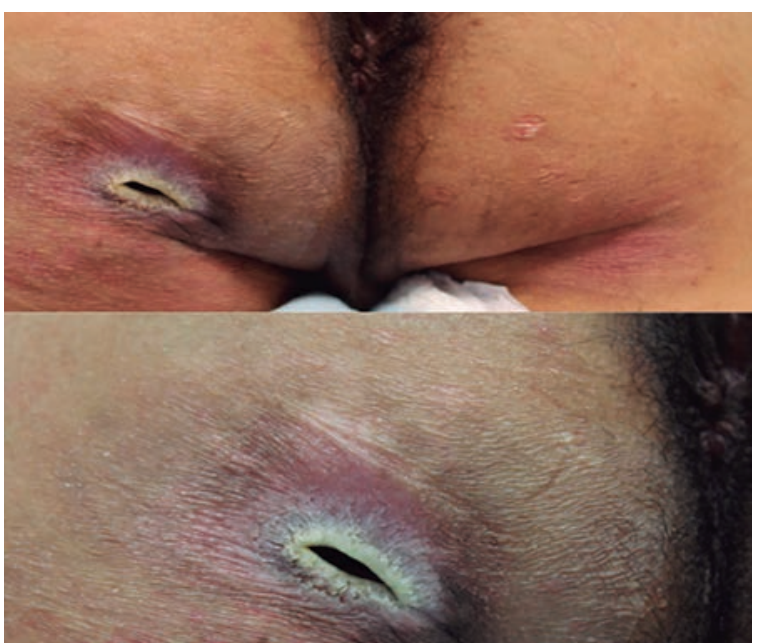

Figura 1. Úlcera isquiática tipo IV con bursa de $6 \times 6$.

sor de fascia lata, bíceps femoral y el colgajo Hamstring, siendo el colgajo Hamstring en V-Y uno de los preferidos'. Las ventajas de este procedimiento son la menor pérdida sanguínea, menor tiempo quirúrgico y una circulación más segura del área isquiática ${ }^{10}$.

El colgajo Hamstring nace de los principios utilizados 1949 por Blocksma et al. para el cierre de una isquiectomía radical por medio de un muñón del bíceps femoral. En los años sesenta se empieza a generalizar la noción de utilizar el bíceps femoral como colgajo de rotación. Hurteau et al., en 1981, confeccionaron un colgajo en isla adicionando el músculo semimembranoso y semitendinoso, en forma de V-Y, y es el primero en utilizar toda la musculatura de esta zona ${ }^{11}$. Posteriormente, en 1999, Janos Jósvay modificó el patrón clásico de V-Y, ya que reportaba una cicatrización residual en la región posterior, realizando solo un corte medial respetando la cara lateral del triángulo propuesto por Hurteau; se logra así menor tensión al cierre y mejor cicatrización ${ }^{12}$.

Se han propuesto otras modificaciones más recientes, como la descrita por Burm, quien propone utilizar la inserción tenomuscular del aductor mayor, así como desinsertar la cabeza larga del bíceps femoral y el músculo semitendinoso, que son separados y avanzados hasta la tuberosidad isquiática, sin desinsertar el tendón distal del bíceps femoral y el músculo semitendinoso ${ }^{13}$.

El objetivo del trabajo fue describir el uso del colgajo Hamstring, poco reportado en la literatura nacional, así como la modificación que aplicamos; y realizar una breve revisión de los conceptos anatómicos claves.

\section{CASO CLÍNICO}

Se presenta el caso de un paciente de sexo masculino de 23 años con diagnóstico de úlcera isquiática izquierda. Cuenta con los siguientes antecedentes patológicos de importancia: mielomeningocele al nacimiento, así como cierre de mielomeningocele a los 8 días de vida, paraplejía secundaria a proceso de tubo neural, reparaciones quirúrgicas de pie equino varo derecho hace 22 y 18 años.

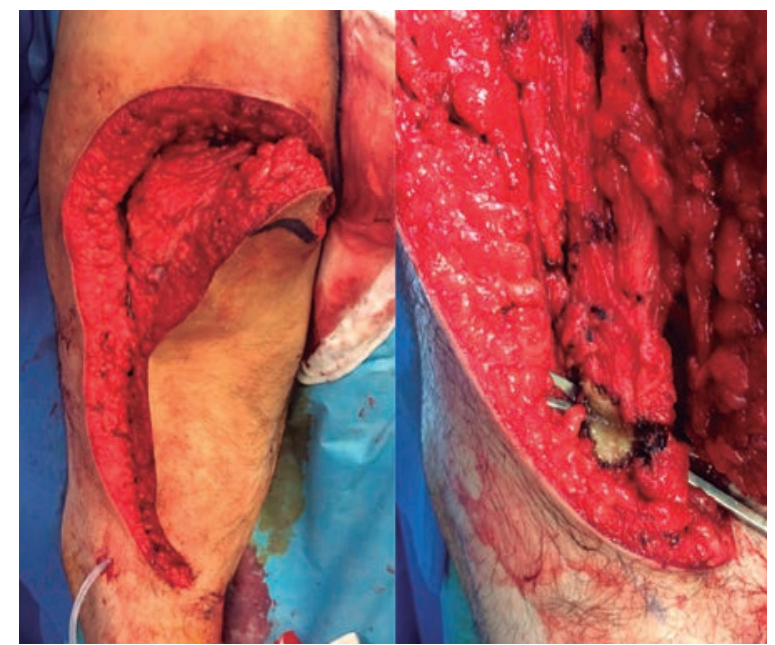

Figura 2. Resección completa de la bursa y remodelación de la tuberosidad isquiática. Se observa el levantamiento del colgajo musculocutáneo y músculos bíceps femoral, semimembranoso y semitendinoso respetando en todo momento los vasos en región poplítea.

Inicia su padecimiento 2 años previos a nuestra valoración presentando úlcera isquiática derecha la cual fue tratada con múltiples tratamientos médicos, apósitos especializaos y lavados quirúrgicos con mala respuesta y profundización.

A la exploración física presentaba una úlcera isquiática tipo IV (según la clasificación de Shea) con compromiso del isquion con bursa periférica de $6 \times 6 \mathrm{~cm}$ y con tejido desvitalizado en los márgenes, sin datos de infección activa (Figura 1).

Técnica quirúrgica: previa desbridación quirúrgica, se realizó incisión lateral y con avance hacia región cefálica y medial, se realiza resección completa de la bursa y remodelación de la tuberosidad isquiática, levantamiento de colgajo musculocutáneo posterior izquierdo con los músculos bíceps femoral, semimembranoso y semitendinoso, realizando desinserción parcial de su inserción distal, protegiendo en todo momento los pedículos vasculares en región poplítea. Se realizó fijación del colgajo hacia el raquis púbico superior y la pared anterior utilizando una sutura de anclaje de material no absorbible para proporcionar cobertura completa (Figuras 2 y 3 ).

Cursa posoperatorio con buena evolución siendo egresado a las 24 horas con seguimiento por consulta externa. A los 7 días presentó dehiscencia parcial en tercio proximal de herida quirúrgica la cual se manejó de forma conservadora inicialmente con la aplicación de apósitos especializados y lavados, más cierre por segunda intención a las 4 semanas (Figura 4).

Actualmente el paciente se encuentra con buena evolución clínica a los 8 meses de posoperatorio, sin datos de dehiscencia o recidiva de úlcera por presión (Figura 5).

\section{DISCUSIÓN}

El colgajo Hamstring está constituido principalmente en el uso de los músculos del plano posterior del muslo: el bíceps femoral (BF), el músculo semimembra- 


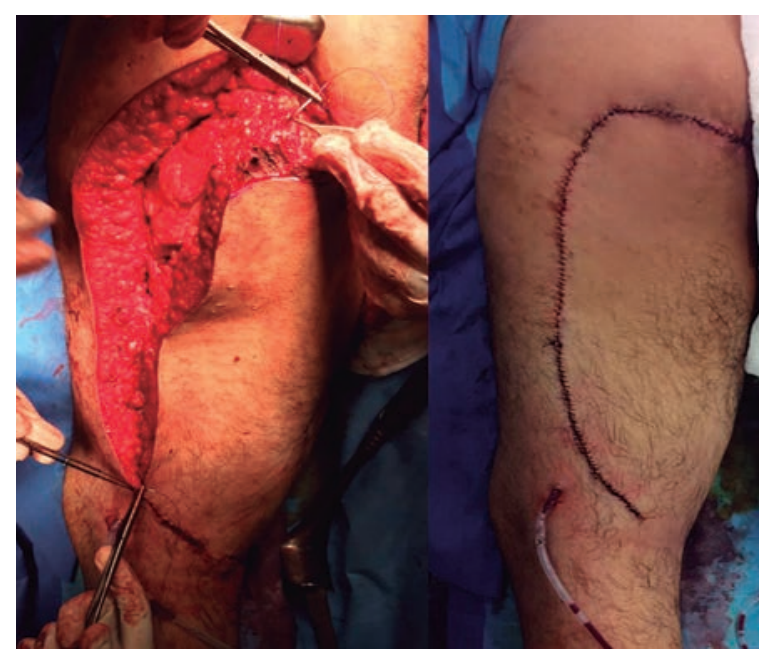

Figura 3. Fijación del colgajo hacia el raquis púbico sin presentarse datos de tensión.

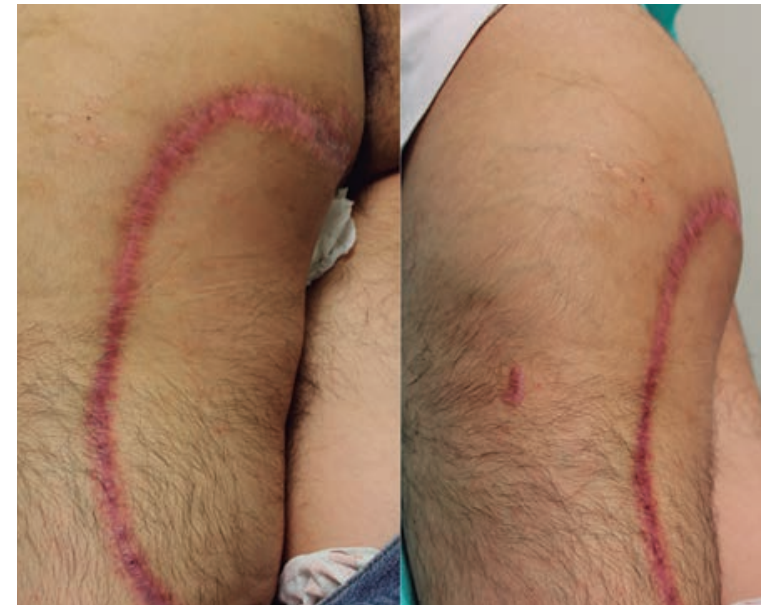

Figura 5. Evolución a los 8 meses de posoperatorio sin observarse recidiva.

noso (MSM) y semitendinoso (MST $)^{14}$. Los tres elementos nacen de la tuberosidad isquiática y músculos Hamstring. Por otro lado, la cabeza corta del BF tiene su origen a lo largo de la línea áspera del fémur uniéndose con la cabeza larga en un tendón común que se inserta en la cabeza del peroné. Los músculos ST y SM se insertan en el cóndilo medial de la tibia, siendo este último el que se ubica medialmente ${ }^{15}$.

La irrigación del músculo BF consta de un pedículo dominante dependiente de la arteria femoral profunda y un pedículo menor que se origina de una rama perforante de esta misma arteria. Ramas colaterales de la arteria genicular también contribuyen en su irrigación menor. El músculo SM tiene como pedículo dominante a ramas perforantes de la arteria femoral profunda y ramas de la arteria glútea inferior. El músculo ST, al igual que sus predecesores, también se irriga a partir de un pedículo dominante de la arteria femoral profunda y sus pedículos menores dependen de la arteria glútea inferior ${ }^{16}$.

Este tipo de colgajo se basa en la versatilidad de la irrigación del muslo posterior y en la capacidad de movilización de los pedículos del tercio inferior de dicho

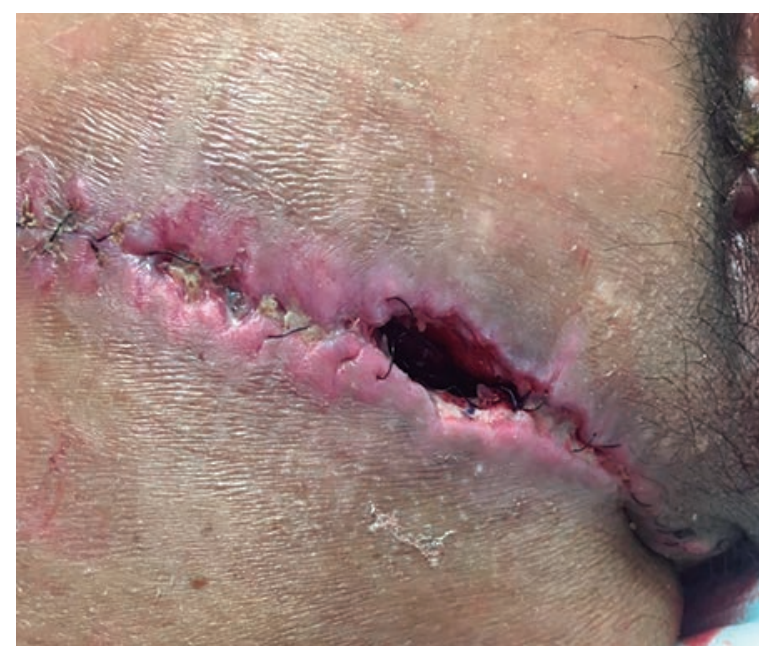

Figura 4. Dehiscencia en región proximal a los 7 días de posoperatorio sin presentar infección.

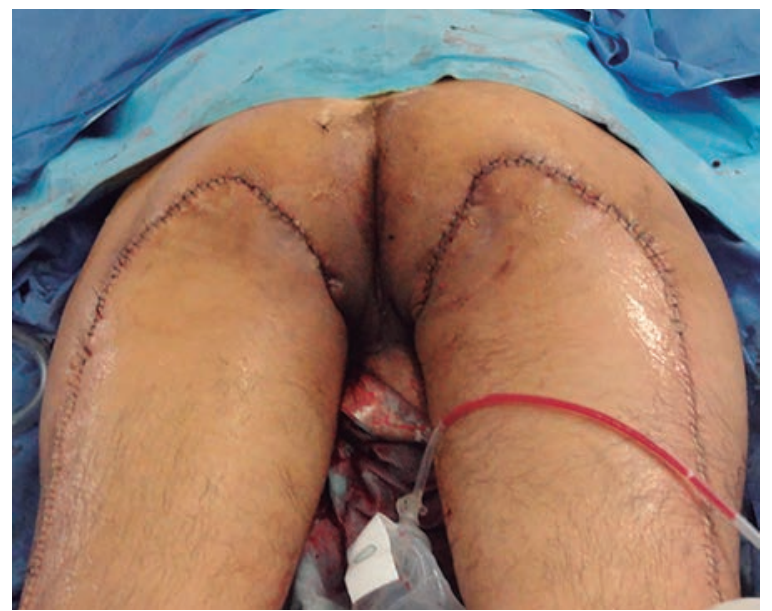

Figura 6. Colgajo modificado de Hamstring bilateral con nuestra técnica para paciente con ulcera isquiática bilateral.

compartimento. La base superior se extiende transversalmente al nivel del pliegue glúteo y el ápice está ubicado justo por encima de la fosa poplítea. Los músculos se dividen en la unión musculotendinosa y se movilizan desde abajo hacia su origen. Los pedículos distales se ligan y los músculos se giran sobre el pedículo proximal. El colgajo es generalmente de 8 a $10 \mathrm{~cm}$ de ancho y puede alcanzar hasta $35 \mathrm{~cm}$ de largo. Proporciona un buen avance ya sea en como colgajo tipo VYo en avance superior, obteniéndose la posibilidad de hacer este colgajo de forma bilateral obteniéndose buena simetría ${ }^{17}$ (Figura 6).

Para el éxito de este tipo de colgajos es importante destacar la necesidad de contar con personal bien entrenado para el control posoperatorio y nutriciona ${ }^{18}$. El manejo posoperatorio requiere un alto nivel de cumplimiento del paciente y sus familiares, sin el cual los buenos resultados a largo plazo no se lograrán ${ }^{19}$. Se ha reportado que el período de mayor probabilidad para recurrencia es entre los primeros 15 a 22 meses $^{20}$. Se destaca la viabilidad del colgajo Hamstring para la reconstrucción de la región isquiática por su bajo número de complicacio- 
TABLA 1. Complicaciones y recurrencias

\begin{tabular}{|c|c|c|c|c|c|}
\hline Autor & \multicolumn{2}{|c|}{ Complicaciones } & Recurrencia & $\mathrm{N}^{\circ}$ de pacientes & Seguimiento \\
\hline Hurteau et al. 1981 & \multicolumn{2}{|l|}{$0 / 10(0 \%)$} & $0 / 10(0 \%)$ & 10 & 3-15 meses \\
\hline \multirow[t]{5}{*}{ Cope et al. 1995} & Total & $7 / 37(18,9 \%)$ & $7 / 37(18,9 \%)$ & 37 & meses \\
\hline & Necrosis parcial & $2 / 37(5,4 \%)$ & & & \\
\hline & Hematoma & $2 / 37(5,4 \%)$ & & & \\
\hline & Infección & $1 / 37(2,7 \%)$ & & & \\
\hline & Ruptura superficial & $2 / 37(5,4 \%)$ & & & \\
\hline Jósvay, Donáth. 1999 & \multicolumn{2}{|l|}{$0 / 11$} & $0 / 11$ & & NA \\
\hline Tavakoli et al. 1999 & \multicolumn{2}{|l|}{$1 / 19(5,3 \%)$} & NA & & meses \\
\hline Watier et al. 2000 & \multicolumn{2}{|l|}{$9 / 38(23,7 \%)$} & $11 / 38(28,9 \%)$ & & NA \\
\hline
\end{tabular}

nes y su menor riesgo de recidivas que otro tipo de colgajos $^{21-23}$. Para fundamentar esto se realizó una revisión encontrando series como la de Hurteau et al. ${ }^{11}$ realizada en 1981, quienes incluyeron 10 casos que no presentaron ninguna complicación ni recurrencia. En otra serie realizada en 1995 , Cope et al. ${ }^{24}$ incluyeron a 37 pacientes de los que presentaron complicaciones $7(18,9 \%)$, que incluían necrosis parcial ( 2 casos), hematoma ( 2 casos), infección (1 caso), ruptura superficial ( 2 casos), así como recurrencia otros $7(18,9 \%)$ pacientes (Tabla 1). En la serie de Jósvay y Donáth ${ }^{12}$, en 1999 , se incluyeron 10 pacientes que no presentaron ninguna complicación ni recurrencia. En la serie de Tavakoli et al. ${ }^{20}$ se incluyeron 19 pacientes en el estudio y reportaron solo $1(5,3 \%)$ con complicación no especificada y recurrencia de $41,4 \%$. O la serie de Watier et al., quienes incluyeron 38 pacientes en su estudio, presentando complicaciones en $9(23,7 \%)$ de ellos y recurrencias 11 (28,9\%).

\section{CONCLUSIÓN}

El colgajo tipo Hamstring sigue siendo una opción de tratamiento para el manejo de úlceras isquiáticas. En centros en donde no se tenga la opción de microcirugía, se propondría el uso de este tipo de colgajo por cirujanos plásticos bien entrenados, adhiriéndolos a la gama de colgajos locales y de avance para un área sumamente complicada.

\section{BIBLIOGRAFÍA}

1. Jordan SW, De la Garza M, Lewis Jr VL, Two-stage treatment of ischial pressure ulcers in spinal cord injury patients: Technique and outcomes over 8-years, J Plast Reconstr Aesthet Surg. 2017 Jul;70(7):959-966.

2. Llanos $S$, et al. Colgajo miocutáneo de glúteo mayor en isla con cierre $\mathrm{V}$ en Y para la cobertura de úlceras isquiáticas. Cir. Plást. Iberlatinamer. Vol. 32 - No 1 Enero - Febrero - Marzo 2006/Pag. 41-48.

3. Anders J, et al. Decubitus ulcers: pathophysiology and primary prevention. Dtsch Arztebl Int 2010;107:371-81-quiz 382.

4. Middleton JW, Lim K, Taylor L, et al. Patterns of morbidity and rehospitalisation following spinal cord injury. Spinal Cord. 2004;42:359-367.

5. Bamba R. et al. Flap Reconstruction for Pressure Ulcers: An Outcomes Analysis. Plast Reconstr Surg Glob Open. 2017

6. Purcel et al. Pelvic Floor Reconstruction Utilizing a Residual Hamstring Rotational Flap Following Traumatically Induced Subtotal Hemipelvectomy in a Combat Blast Casualty: A Case Report. Military Medicina, Vol. 181, September 2016.

7. Cushing CA, Phillips LG. Evidence-based medicine: pressure sores. Plast Reconstr Surg. 2013 Dec;132(6):1720-32.

8. Marci M, Battaglia S, Marchese S, Intagliata E, Spartaro, Vecchio R. Surgical reconstructive procedures for treatment of ischial, sacral and trochanteric pressure ulcers. G Chir. 2015 May-Jun; 36(3): 112-6.

9. Lin $\mathrm{H}$, et al. Treatment of ischial pressure sores using a modified gracilis myofasciocutaneous flap. J Reconstr Microsurg. 2010 Apr;26(3):153-7

10. Kuo PJ, Chew KY, Kuo YR, et al. Comparison of outcomes of pressure sore reconstructions among perforator flaps, perforator-based rotation fasciocutaneous flaps, and musculocutaneous flaps. Microsurgery. 2014;34:547-53.

11. Hurteau JE, Bostwick J, Nahai F, Hester R, Jurkiewics MJ. V-Y advancement of hamstring musculocutaneous flap for coverage of ischial pressure sores. Plast. Reconstru. Surg. 68. 539, 1981

12. Josvay J., Donath Antal. Modified Hamstring Musculocutaneous flap for the coverage of ischial pressure sores. Plast Reconstr Surg. 1999 May; 103(6):1715-8.

13. Burm JS1, Hwang J, Lee YK. A New Option for the Reconstruction of Primary or Recurrent Ischial Pressure Sores: Hamstring-Adductor Magnus Muscle Advancement Flap and Direct Closure. Ann Plast Surg. 2018 Jan 5
14. Battermann N, Appell HJ, Dargel J, Koebke J. An anatomical study of the proximal hamstring muscle complex to elucidate muscle strains in this region. Int J Sports Med. 2011 Mar;32(3):211-5

15. Van der Made AD, Wieldraaijer T, Kerkhoffs GM, Kleipool RP, Engebretsen L, van Dijk CN, Golanó P. The hamstring muscle complex. Knee Surg Sports Traumatol Arthrosc. 2015 Jul;:23(7):2115-22

16. Woodley SJ, Mercer SR. Hamstring Muscles: Architecture and Innervation. Cells Tissues Organs 2005; 179:125-141

17. Stephen J. Mathes, Foad Nahai, Mathes. Regional Flaps: Anatomy and Basic Techniques / Thigh. Clinical Atlas Of Muscle And Musculocutaneous Flaps. Ed 1979. Ed. Mosby

18. Keys KA, Daniali LN, Warner KJ, Mathes DW. Multivariate predictors of failure after flap coverage of pressure ulcers. Plast Reconstr Surg. 2010 Jun;125(6):1725-34

19. Niederhauser A, Lukas CV, Parker V, Ayello EA, Zulkowski K, Berlowitz $D$. Comprehensive programs for preventing pressure ulcers: a review of the literature. Advances in skin \& wound care. 2012;25(4):167-88.

20. Tavakoli K, Rutkowski S, Cope C, et al. Recurrence rates of ischial sores in para-and tetraplegics treated with hamstring flaps: An 8-year study. Br J Plast Surg. 1999;52:476-479

21. Evans G, Dufresne C, Manson P. Surgical correction of pressure ulcers in an urban center: Is it efficacious? Adv Wound Care 1994; 7:40-46

22. Chiu YJ, Liao WC, Wang TH, Shih YC, MaH, Lin CH, Wu SH, Perng CK. ARetrospective Study: Multivariate Logistic Regression Analysis of the Outcomes after Pressure Sores Reconstruction With Fasciocutaneous, Myocutaneous and Perforator Flaps. J Plast Reconstr Aesthet Surg. 2017 Aug;70(8):1038-1043

23. Sameem M, Au M, Wood T, Farrokhyar F, Mahoney J. A systematic review of complication and recurrence rates of musculocutaneous, fasciocutaneous, and perforator-based flaps for treatment of pressure sores. Plastic and reconstructive surgery. 2012;130(1):67e-77e

24. Cope C, Barry P, Hassall M, Barnett R, Richards M, Vandervord J. V-y advancement hamstring myocutaneous island flap repair of ischial pressure ulcers. ANZ Journal of Surgery. junio de 1995;65(6):412-6. 